\title{
Experimental Atom-To-Solid $L$-Level Shifts for Ce, Sm, Gd, and Er
}

\author{
G. Materlik \\ Hamburger Synchrotronstrahlungslabor HASYLAB, Deutsches Elektronen-Synchrotron DESY, \\ D-2000 Hamburg 52, Germany \\ and \\ B. Sonntag and M. Tausch \\ II. Institut für Experimentalphysik, Universität Hamburg, D-2000 Hamburg 50, Germany \\ (Received 18 July 1983)
}

\begin{abstract}
The x-ray $L$ absorption spectra were measured for the atomic rare-earth elements $\mathrm{Ce}, \mathrm{Sm}, \mathrm{Gd}$, and $\mathrm{Er}$. From the energy positions of well resolved absorption lines at threshold in comparison to the corresponding solid-state spectra the atom-to-metal shifts of the core-level binding energies have been determined. For Sm and Er which undergo a valence change on formation of the solid the shifts differ by more than $6 \mathrm{eV}$ from those of Ce and Gd which have the same configuration in the atom and the solid.
\end{abstract}

PACS numbers: 78.70.Dm, 32.30.Rj, 71.50.+t

Shifts of core-level binding energies of atoms in different chemical environments reflect changes in valence orbitals and in the valence configuration and differences in the relaxation processes. The fingerprint character of these shifts explains the considerable experimental and theoretical effort devoted to the investigation of this subject., ${ }^{1,2}$ Especially, the atom-to-metal shifts of the rare-earth elements recently have attracted much interest. This interest has been stimulated by the valence fluctuations detected in pure rare-earth elements and rare-earth compounds. ${ }^{3-5}$ One of the major problems encountered in this field is the lack of experimental data on free metal atoms in the $x$-ray region. There are almost no x-ray-absorption, x-ray-emission, and $x$-ray-photoemission data on free metal atoms, from which core-level binding energies, transition probabilities, core-hole lifetimes, and indications of the importance of manyelectron effects can be obtained. In addition to being important for atomic physics, all these values are necessary for a thorough assessment of the influence of the chemical environment. Oscillations in the differential optical oscillator strength ${ }^{6}$ or, more important, multiple-electron excitations ${ }^{7}$ can cause structures in the absorption spectra above inner-shell thresholds. These effects have to be considered in the analysis of the $\mathrm{x}-\mathrm{ray}-\mathrm{abs}$ rption near-edge structure $(\mathrm{XANES})^{8}$ and the extended $\mathrm{x}-\mathrm{ray}-\mathrm{abs}$ orption fine structure (EXAFS) ${ }^{9}$ of solids.

The high temperatures required for the preparation of free rare-earth atoms and their low absorption cross sections render these experiments extremely difficult. Since a lot of basic information can be obtained from absorption spectra, we measured the $L$ absorption spectra of a representative set of atomic rare-earth elements, Ce, Sm, Gd, and Er.

The measurements were done at the $x$-ray spectrometer ROEMO at the Hamburger Synchrotron Radiation Laboratory (HASYLAB). We have used a nondispersive Si double-crystal monochromator optimized for small harmonic content. ${ }^{10}$ To balance high energy resolution and higher-order contamination, (111) Bragg reflection was used for $\mathrm{Ce}$ and $\mathrm{Sm}$ and (220) Bragg reflection for Gd and Er. The atomic metals were maintained inside a resistence-heated tubular furnace ${ }^{11}$ mounted behind the exit slit of the monochromator. The metal vapors were contained in a Ta tube except for Er where a W tube was used. The temperatures ranged from 1320 to $2500 \mathrm{~K}$, namely, $1320 \pm 50 \mathrm{~K}$ for $\mathrm{Sm}, 2020 \pm 50$ $\mathrm{K}$ for $\mathrm{Er}, 2250 \pm 50 \mathrm{~K}$ for $\mathrm{Gd}$, and $2500 \pm 50 \mathrm{~K}$ for $\mathrm{Ce}$, which according to vapor-pressure data ${ }^{12}$ correspond to pressures between 2 and 16 mbar. The length of the vapor column was approximately $50 \mathrm{~cm}$. Be windows (400 $\mu \mathrm{m}$ thick) separated the vapor region from the surrounding air. A Ne buffer gas ( 20 to $30 \mathrm{mbar}$ ) retarded the escape of the metal atoms from the hot zone of the furnace and prevented them from reaching the $\mathrm{Be}$ windows. Charging the furnace with a sample of $\sim 50 \mathrm{~g}$ made possible stable operation for $\sim 4-6 \mathrm{~h}$. For the exact determination of the atom-to-metal shifts, the spectra of the rare-earth metals were recorded simultaneosuly with the vapor spectra by use of three ionization chambers in a row. The metal sample was placed between the first and the second and the vapor between the second and third chambers. In some cases metal runs were taken immediately before or after each 
vapor measurement.

The $L_{3}$ absorption spectra of atomic and metallic $\mathrm{Ce}, \mathrm{Sm}, \mathrm{Gd}$, and $\mathrm{Er}$ are presented in Fig. 1. Since the $L_{2}$ spectra are very similar to the $L_{3}$ spectra they are not shown. The prominent white lines at the threshold of the atomic $L_{2}$ and $L_{3}$ absorption are attributed to $2 p_{1 / 2,3 / 2} \rightarrow 5 d$ transitions. As demonstrated in Fig. 1 these lines can be very well fitted by a single Lorentzian. Autoionization, which could cause an asymmetric line shape, ${ }^{13}$ does not contribute significantly to the decay of the $2 p$ core hole. The onset of transitions to higher excited states manifests itself

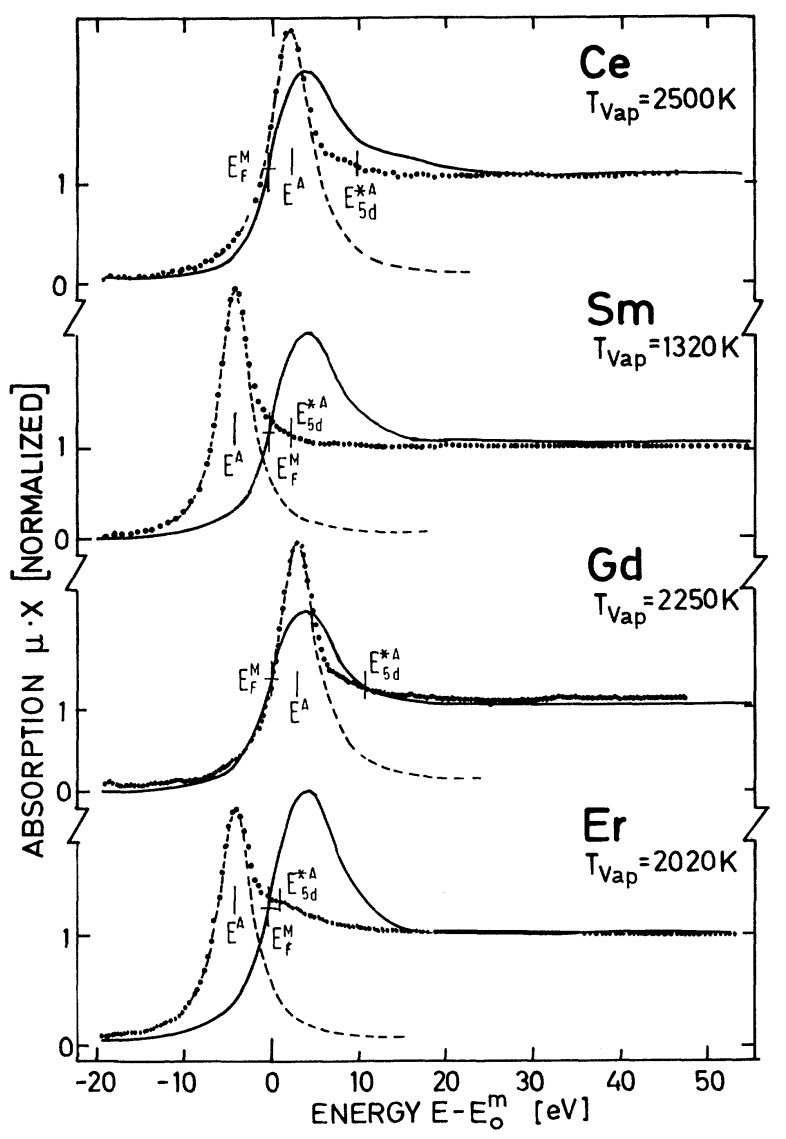

FIG. 1. $L_{3}$ absorption spectra of atomic (dotted lines) and metallic (solid lines) $\mathrm{Ce}, \mathrm{Sm}, \mathrm{Gd}$, and $\mathrm{Er}$. A background, determined by extrapolating the absorption spectrum below the $2 p$ threshold towards higher energies by a straight line, has been subtracted. The positions of the Fermi levels $E_{\mathrm{F}}{ }^{M}$, the atomic $5 d$ excitation energies $E^{A}$, and the ionization energies $E_{5 d^{* A}}$ are indicated. The dashed line gives a Lorentzian absorption profile. The edge heights are normalized. The inflection point $E_{0}{ }^{m}$ of the absorption edge of the solid-state spectrum is chosen as the origin of the energy scale. on the high-energy side in the deviation of the experimental curve from the Lorentzian. A break in the atomic absorption spectrum, which is clearly to be seen $\sim 3 \mathrm{eV}$ above the center of the white line, is most likely due to $2 p \rightarrow 6 d$ transitions. On the formation of the solid the white lines are shifted and broadened by the interaction with the neighboring atoms. Materlik, Müller, and Wilkins ${ }^{14}$ demonstrated that the white line and the near-edge structure can be well described by a single-particle band-structure calculation. For $\mathrm{Ce}$ and $\mathrm{Gd}$ which have the same $4 f^{n}$ configuration $\left(\mathrm{Ce} 4 f^{1}, \mathrm{Gd} 4 f^{7}\right)$ in the atom and in the solid the centers of the white lines almost coincide (1-2-eV shift). In contrast to this, for $\mathrm{Sm}$ and Er, which undergo a change of the $4 f^{n}$ configuration on solidification $\left(\operatorname{Sm~} 4 f^{6} \rightarrow 4 f^{5} ; \operatorname{Er} 4 f^{12}\right.$ $\rightarrow 4 f^{11}$ ) the peak of the white line is shifted towards higher energies by about $8 \mathrm{eV}$.

The $L_{1}$ spectra of the atomic and metallic samples are given in Fig. 2. The atomic spectra display a clearly discernible line at threshold due to $2 s \rightarrow 6 p$ transitions. The strength of this line is comparable to the strength of the $2 s \rightarrow \epsilon p$ continuum transitions. Therefore the line is harder to disentangle from the transitions to higher excited states than the $2 p \rightarrow 5 d$ absorption lines dis cussed above. In the solid the hybridization of the outer $s, p$, and $d$ symmetric orbitals changes the spectra at threshold completely. The metal spectra show a steep rise at threshold followed by a further increase of the cross section towards the maximum located approximately 20 $\mathrm{eV}$ above the onset. The experimental spectrum is well reproduced by one-electron band-structure calculations. ${ }^{14}$ The shoulder at threshold and the dominant maximum at higher energies reflect the structure of the $p$-projected density of conduction-band states. Again the atoms undergoing a valence change ( $\mathrm{Sm}, \mathrm{Er}$ ) on solidification and those which do not (Ce, Gd) are easily distinguished. For $\mathrm{Ce}$ and $\mathrm{Gd}$ the steep rise at threshold of the metal spectra almost coincides with the rise of the atomic cross section whereas for Sm and Er they are separated by more than $5 \mathrm{eV}$.

From the area under the Lorentzian lines the ratio of the cross sections $\sigma\left(2 p_{3 / 2} \rightarrow 5 d\right) / \sigma\left(2 p_{1 / 2}\right.$ $\rightarrow 5 d$ ) has been determined to be $2.1 \pm 0.1$. Within the error limits this ratio agrees with the statistical ratio of 2 and indicates only small differences between the respective matrix elements. The cross section $\sigma(2 s \rightarrow 6 p)$, determined in an analogous way, is approximately a factor of 6 


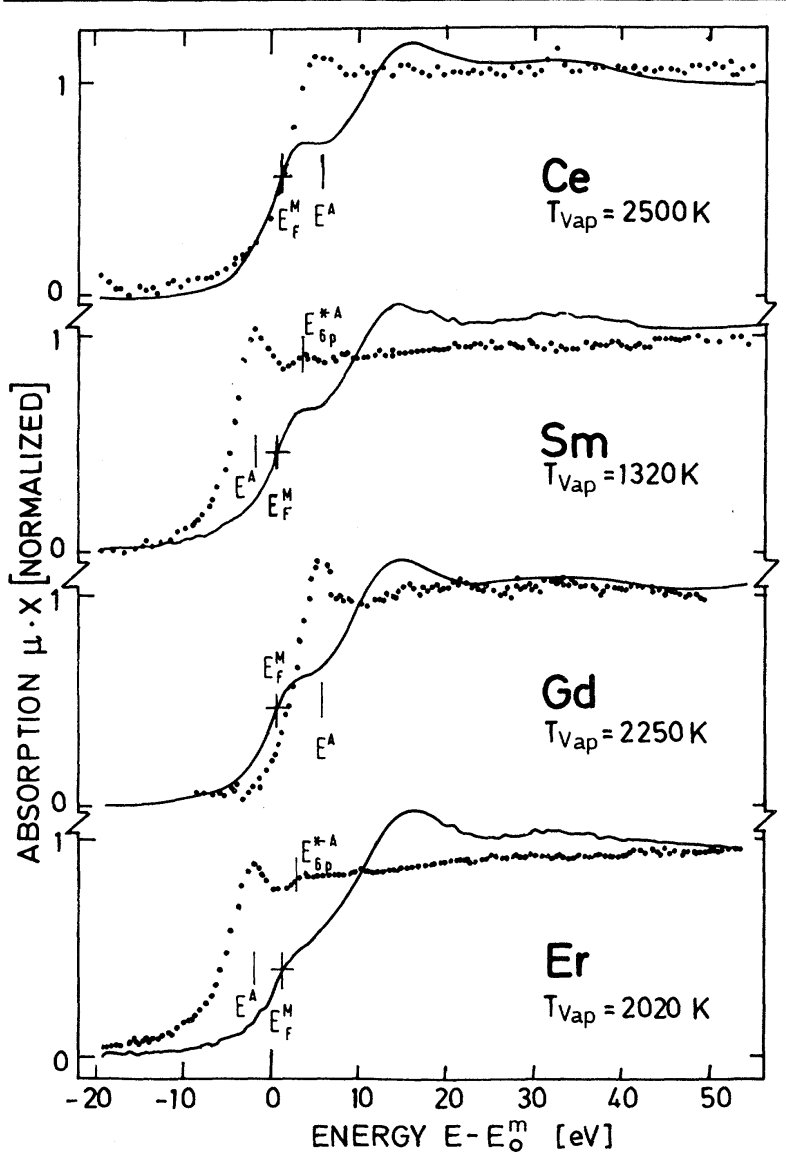

FIG. 2. $L_{1}$ absorption spectra of atomic (dotted lines) and metallic (solid lines) $\mathrm{Ce}, \mathrm{Sm}, \mathrm{Gd}$, and $\mathrm{Er}$. A background, determined by extrapolating the absorption spectrum below the $2 s$ threshold towards higher energies by a straight line, has been subtracted. The positions of the Fermi levels $E_{\mathrm{F}}{ }^{M}$, the atomic $6 p$ excitation energies $E^{A}$, and the ionization energies $E_{6 p}{ }^{* A}$ are indicated. The edge heights are normalized. The inflection point $E_{0}{ }^{m}$ of the absorption edge of the solid-state spectrum is chosen as the origin of the energy scale.

smaller than o $\left(2 p_{3 / 2} \rightarrow 5 d\right)$.

From the comparison of the experimental and the calculated spectra $\mathrm{a}^{14}$ the metal core-level binding energies referred to the Fermi level $E_{2 s, \mathrm{~F}}{ }^{M}, E_{2 p, \mathrm{~F}}{ }^{M}$ have been inferred. ${ }^{15}$ The positions of the maxima of the atomic absorption lines are used to determine the $2 p \rightarrow 5 d$ and $2 s$ $\rightarrow 6 p$ excitation energies $E_{2 p \rightarrow 5 d}{ }^{A}$ and $E_{2 s \rightarrow 6 p}{ }^{A}$. The energy differences

$$
\Delta E=E_{\mathrm{ex}}{ }^{\boldsymbol{A}}-E_{c \mathrm{~F}}{ }^{M}
$$

between the atomic excitation energies $E_{\mathrm{ex}}{ }^{A}$ and the metal core-level binding energies $E_{c \mathrm{~F}}{ }^{M}$ are
TABLE I. Experimental energy separation $\Delta E_{L}$ $=\boldsymbol{E}_{\text {ex }}{ }^{A}-E_{c \mathrm{~F}}{ }^{M}$ and experimental $\delta E_{L}$ and theoretical (Refs. 1 and 2) $\delta E^{\text {calc }}$ atom-to-metal core-level binding-energy shifts. The atomic $5 d$ and $6 p$ binding energies $E_{5 d} * A$ and $E_{6 p} * A$ are given in the following sequence: (Ref. 16 value) $/\left(Z+1\right.$ model value). $\delta E_{L_{2,3}}$ and $\delta E_{L_{1}}$ were calculated with values from Ref. 16 and from the $Z+1$ model, respectively. Note that the tabulated error only includes the uncertainity to determine $E_{\text {ex }}{ }^{A}$ and $E_{0}{ }^{m}$ from the experimental data. All energies are in electronvolts.

\begin{tabular}{|c|c|c|c|c|}
\hline & $\mathrm{Ce}$ & $\mathrm{Sm}$ & $\mathrm{Gd}$ & Er \\
\hline$\Delta E_{L_{3}}$ & $2.6 \pm 0.3$ & $-3.8 \pm 0.3$ & $2.8 \pm 0.3$ & $-3.7 \pm 0.3$ \\
\hline$E_{5 d} * A$ & $7.6 / 7.4$ & $6.3 / 6.7$ & $7.6 / \bullet$ & $5.2 / 5.7$ \\
\hline$\delta E_{L_{3}}$ & $10.2 \pm 0.3$ & $2.5 \pm 0.3$ & $10.4 \pm 0.5$ & $1.5 \pm 0.3$ \\
\hline$\Delta E_{L_{2}}$ & $2.6 \pm 0.3$ & $-3.9 \pm 0.3$ & $2.9 \pm 0.3$ & $-3.4 \pm 0.3$ \\
\hline$E_{5 d} * A$ & $7.6 / 7.4$ & $6.3 / 6.7$ & $7.6 / \ldots$ & $5.2 / 5.7$ \\
\hline$\delta \boldsymbol{E}_{L_{2}}$ & $10.2 \pm 0.3$ & $2.4 \pm 0.3$ & $10.5 \pm 0.3$ & $1.8 \pm 0.3$ \\
\hline$\Delta E_{L_{1}}$ & $5.3 \pm 0.6$ & $-2.5 \pm 0.5$ & $4.7 \pm 0.4$ & $-3.6 \pm 0.7$ \\
\hline$E_{6 p}{ }^{* A}$ & $\cdots / \cdots$ & ../ 5.0 & $\ldots / \ldots$ & $\ldots / 4.9$ \\
\hline$\delta E_{L_{1}}$ & . & $2.5 \pm 0.5$ & $\cdots$ & $1.3 \pm 0.7$ \\
\hline$\delta E^{\mathrm{calca}}$ & 9.3 & 0.4 & 8.4 & 0 \\
\hline$\delta E^{\mathrm{calcb}}$ & 9.7 & 1.8 & 9.1 & 1.5 \\
\hline
\end{tabular}

${ }^{\mathrm{a}}$ Ref. 1 .

${ }^{\mathrm{b}}$ Ref. 2 .

listed in Table I. Valence changes on solidification can be well identified with the help of these $\Delta E$ values. In order to compare our energy shift $\Delta E$ with the calculated core-level binding-energy shifts

$$
\delta E=E_{c}{ }^{A}-E_{c \mathrm{~F}^{M}}=E_{\mathrm{ex}}{ }^{A}+E_{c}{ }^{* A}-E_{c \mathrm{~F}}{ }^{M}
$$

we have to correct for the atomic binding energy of a $5 d\left(E_{5 d} * A\right)$ or $6 p\left(E_{6 p} * A\right)$ electron in the presence of a $2 p$ or $2 s$ hole, respectively. Since the $2 s$ and $2 p$ ionization energies cannot be directly read off from the spectra, values for the binding energies $E_{5 d}{ }^{* A}$ were used from atomic relativistic Hartree-Fock calculations ${ }^{16}$ to determine $\delta E_{L_{2}}$ and $\delta E_{L_{3}}$. For $\delta E_{L_{1}}$ no such values were available so that we had recourse to the $Z+1$ model. Therefore in Table I approximate values for $E_{5 d} *^{A}$ and $E_{6 p} *^{A}$ are listed also, which have been obtained from tabulated energy levels of the $Z+1$ atoms. ${ }^{17-19}$ As seen, values obtained from either method show very good agreement. In this context we want to remind the reader that the interaction of the atomic valence electrons results in a multiplet splitting of 0.5 to $1.0 \mathrm{eV},^{17-19}$ which can modify the tabulated shifts correspondingly.

For each element the differences between the $\delta E_{L_{1}}, \delta E_{L_{2}}$, and $\delta E_{L_{3}}$ shifts are within the error 
limits. In view of the discussion above, the atomto-metal core-level-independent binding-energy shifts calculated by Johansson and Martensson ${ }^{2}$ are in good agreement with the experimental values. The values reported from the $a b$ initio calculation by Herbst ${ }^{1}$ also agree reasonably well with our measurements and show, even at these deep core states, only small differences. Both experimental and theoretical values show clearly the strong influence of the atom-to-metal valence change.

We would like to thank Dr. J. F. Herbst for making his calculated atomic binding energies $E_{c} * A$ available to us.

${ }^{1}$ J. F. Herbst, Phys. Rev. Lett. $\underline{49}, 1586$ (1983), and references therein.

${ }^{2}$ B. Johansson and N. Martensson, Phys. Rev. B 21 , 4427 (1980).

${ }^{3}$ H. Launois, M. Rawiso, E. Holland-Moritz, R. Pott, and D. Wohlleben, Phys. Rev. Lett. 44, 1271 (1980).

${ }^{4}$ Valence Fluctuations in Solids, edited by L. M. Falicov, W. Hanke, and M. B. Maple (North-Holland, Amsterdam, 1981).

${ }^{5}$ Valence Instabilities, edited by $\mathrm{P}$. Wachter and H. Boppart (North-Holland, Amsterdam, 1982).

${ }^{6} \mathrm{~S}$. T. Manson and M. Inokuti, in Inner-Shell and $X-R a y$ Physics of Atoms and Solids, edited by D. J.
Fabian, H. Kleinpoppen, and L. M. Watson (Plenum, New York, 1981), p. 273.

${ }^{7}$ W. Malzfeldt, W. Niemann, and P. Rabe, to be published.

${ }^{8}$ See, e.g., M. Belli, A. Scafati, A. Bianconi, S. Mobilio, L. Palladino, A. Reale, and E. Burattini, Solid State Commun. 35, 355 (1980).

${ }^{9}$ See, e.g., G. S. Brown and S. Doniach, in Synchroton Radiation Research, edited by $\mathrm{H}$. Winick and S. Doniach (Plenum, New York, 1980), p. 353.

${ }^{10} \mathrm{G}$. Materlik and V. O. Kostroun, Rev. Sci. Instrum. 51,86 (1980).

${ }^{11}$ R. Bruhn, B. Sonntag, and H. W. Wolff, J. Phys. B 12, 203 (1979).

${ }^{12}$ W. F. Brunner and T. H. Batzer, Practical Vacuum Techniques (Reinhold, New York, 1965).

${ }^{13} \mathrm{~A}$. F. Starace, in Corpuscles and Radiation in Matter 1, Handbuch der Physik Vol. 31, edited by W. Mehlhorn (Springer-Verlag, Berlin, 1982).

${ }^{14}$ G. Materlik, J. F. Müller, and J. W. Wilkins, Phys. Rev. Lett. 50, 267 (1983).

${ }^{15}$ With respect to the inflection point of the experimental spectra, the following values were used for $E_{c \mathrm{~F}}{ }^{M}$ $-E_{0}{ }^{m}$ given in electronvolts in the sequence $L_{3}, L_{2}$, $L_{1}$ : Ce: $-0.3,-0.3$, +0.5; Sm: $-0.5,-0.5$, +0.6; Gd: $-0.4,0.4,+0.6$; Er: $-0.3,-0.5,+1.6$.

${ }^{16} \mathrm{~J}$. F. Herbst, private communication.

${ }^{17}$ L. Brewer, J. Opt. Soc. Am. 61, 1101 (1971).

${ }^{18}$ L. Brewer, J. Opt. Soc. Am. $\underline{61}, 1666$ (1971).

${ }^{19}$ W. C. Martin, R. Zalubas, and L. Hagan, Atomic Energy Levels - The Rare Earth Elements, National Bureau of Standards Publication No. NSRDS-NBS 60 (U.S. GPO, Washington, D.C., 1978). 